\title{
Referral Pattern to Neurosurgeons in a tertiary care teaching Hospital
}

\author{
M. Srinivas ${ }^{1}$, S. Satish Kumar ${ }^{1}$, Venkata Ramya Bola ${ }^{2}$, Ranabir Pal ${ }^{3}$, \\ Radhika Gorantla $^{4}$, M. Jahnavi ${ }^{5}$, B.V. Subramanyam ${ }^{6}$, Luis Rafael \\ Moscote-Salazar ${ }^{7}$, R. Lakshman Kumar ${ }^{5}$, Amrita Ghosh ${ }^{8}$, Amit \\ Agrawal $^{5}$ \\ ${ }^{1}$ Department of Emergency Medicine, Narayana Medical College Hospital, Chinthareddypalem, \\ Nellore, AP, INDIA \\ ${ }^{2}$ Department of Management Information System, Narayana Medical College Hospital, \\ Chinthareddypalem, Nellore, AP, INDIA \\ ${ }^{3}$ Department of Community Medicine, ESIC Medical College and Hospital, Faridabad, INDIA \\ ${ }^{4}$ Department of Hospital Administration, Narayana Medical College Hospital, \\ Chinthareddypalem, Nellore, AP, INDIA \\ ${ }^{5}$ Department of Neurosurgery, Narayana Medical College Hospital, Chinthareddypalem, \\ Nellore, AP, INDIA \\ ${ }^{6}$ Department of Forensic Medicine, Narayana Medical College Hospital, Chinthareddypalem, \\ Nellore, AP, INDIA \\ ${ }^{7}$ Cartagena Neurotrauma Research Group, University of Cartagena, Cartagena de Indias, \\ COLOMBIA \\ ${ }^{8}$ Department of Biochemistry, Medical College, CALCUTTA
}

\begin{abstract}
Background: Due to lack of pre-hospital care, direct admission of traumatic brain injury (TBI) patients to dedicated neurosurgical department remains difficult in many parts of world. Objective: The present pilot study was conducted to understand the referral pattern of patients to the neurosurgery department and to develop an algorithm to stratify the spectrum of cases as per the severity of condition and possibility of direct neurosurgical care. Materials and Methods: Details of referral patterns, demographic and clinical profile, management and outcome of consecutive TBI cases admitted in the Department of Neurosurgery were collected with predesigned proforma. Results: Of total 55 cases, 40 (73\%) were males; 26 (47\%) between 36-55 years age; 26 (47\%) referred directly to the center; 24 (44\%) self-referred; 34 (62\%) referred from Emergency department; mostly (32, 58\%) admitted after 12PM; 22 (40\%) admitted for 16-30 days. In the analysis of investigations, 37(67\%) cases had CT,
\end{abstract}


15 (27\%) had MRI, 4 (7\%) had X-ray, 1 (2\%) undergone all three investigations, while 8 (15\%) didn't have any; mostly $(42,76 \%)$ cases were referred for the management of TBI and $38(69 \%)$ required operative interventions. Conclusion: Our findings strongly suggest that referral of TBI cases need improvement in this part of country where hindrances delay TBI cases to reach dedicated neurological intensive care leading to delay of committed care and optimization of strategy for reduction of case fatality.

Key words: Head Injury, referrals, neurosurgical management, tertiary care hospital

\section{Introduction}

Road traffic accidents remain the single biggest cause of injuries and injury-related mortality, accounting for a quarter of all injury deaths. [1] Worldwide, an estimated 1.3 million people die on roads each year and as many as 78.2 million get injured. Projections indicated that these figures will increase by about 65 percent over the next 20 years, unless there is a new commitment to prevention initiated. This effect of projected increases will be greater in low- and middleincome countries, as they currently account for over 90 percent of all road traffic injury deaths. [2] The problem of road trauma and its growing trend in the low- and middleincome countries is not a new problem and it has previously been described as a neglected global epidemic. [3] worldwide road traffic accidents account for an estimated 50 million injuries. [4] All over the world about 59 percent of road traffic deaths occur among productive age group i.e. young adults between 15 and 44 years. [5] Our center Narayana Medical College Hospital, Nellore, Andhra Pradesh, India, continues to play a pivotal role in the management of head and spinal cord injuriesin this region. Many patients with acute or chronic neurological problems often get admitted under general medicine and other departments. Some with other primary diagnosis may also develop neurological complication due to course of illness or as a part of complication. This is a descriptive cross-sectional study of a Head Injury (HI) registry was conducted with the aim of establishing the pattern of referrals of $\mathrm{HI}$ for neurosurgery dept. The findings of this study hopefully will provide insights for the neurosurgical management of $\mathrm{HI}$ in the dedicated tertiary care teaching hospitals in South India.

\section{Materials and Methods}

This is a prospective study of consecutive HI patients managed in a neurosurgical dept. at the Narayana Medical College Hospital $(\mathrm{NMCH})$, Nellore, Andhra Pradesh, India between March to October 2017. NMCH, the Centre of excellence is a 1420 bedded tertiary care teaching hospital committed to provide exemplary care to meet or exceed the patient expectations. Each of the referred patients was examined by the Neurosurgeon who attended the referral. The patients were diagnosed clinically with proper history, examination with the help of investigations in required cases. Information regarding the demographic and clinical profile were gathered through a pretested questionnaire. 
The Research Protocol was reviewed and accepted by the Institutional Ethical Committee, NMCH. Our study population included 55 trauma patients whose case sheets of head injury were examined for the referral patterns. Firstly, the cases of head injury presenting primary referral and selfreferral and to the NMCH were sorted. Selfreferral was defined as the cases of head injury that presented directly at the Neurosurgery department and the primary referral was defined as those cases examined initially in another health facility before presenting in $\mathrm{NMCH}$ were labeled the primary referral ones. The sources of referrals of the latter were noted including types of health facilities (private, general hospitals or other tertiary facilities) from which they were referred for neurosurgical care, and the place of origin in Nellore where the trauma occurred. The number of health facilities (one, two and more) earlier visited before arrival in $\mathrm{NMCH}$, Nellore, as well as whether there was any in-house neurosurgical service in any of these facilities was also noted. The reason for the inter-hospital transfers from the other centers was also noted from the referral letters whenever available.

\section{Statistical analysis}

The clinical profile of the cases was transferred to an electronic spreadsheet, and analyzed using the SPSS version 24 (The SPSS Inc, II, USA) and presented here in descriptive statistics including frequencies and proportions, means (standard deviation, SD), 95\% confidence, CI, median (range). Categorical variables were explored for associations with the chi-squared test. An alpha value of $<0.05$ was deemed significant for associations.

\section{The referral pattern of the neurosurgical cases} represented as follows:

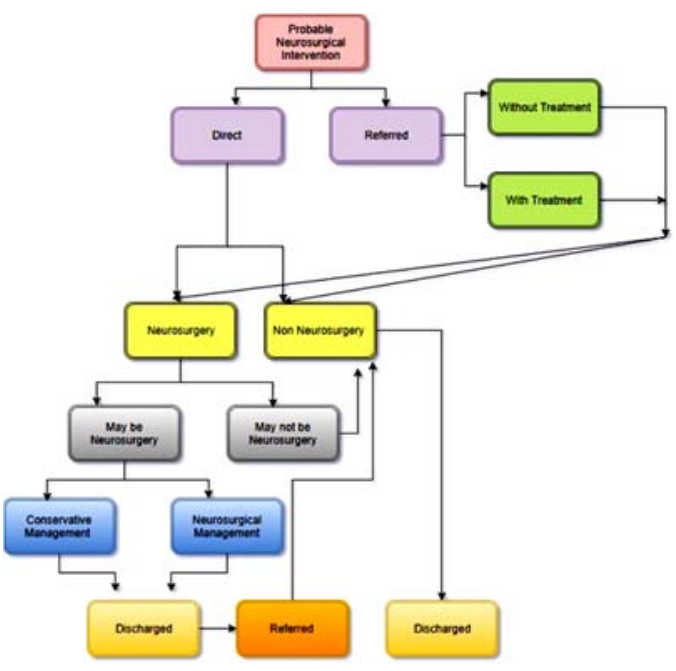

\section{Results}

Fifty five (55) neurosurgery referral cases managed at this tertiary care center were taken up in this study and their clinical data is captured in the registry over period of eight months. In the demographic details, of these 55 cases, $73 \%$ are males and $27 \%$ were females. Only $2 \%$ are less than 18 years of age, $16 \%$ are in the age group 18 -35years, $47 \%$ are under the age group 36-55years and 35\% are above 55 years (Table 1). 
Table 1 - Demographic profile of the Neurosurgery referral cases

\begin{tabular}{|l|l|l|}
\hline Gender & Frequency & Percent \\
\hline F & 15 & $27 \%$ \\
\hline M & 40 & $73 \%$ \\
\hline Age Group & & \\
\hline$<18$ years & 1 & $2 \%$ \\
\hline $18-35$ years & 9 & $16 \%$ \\
\hline $36-55$ years & 26 & $47 \%$ \\
\hline$>55$ years & 19 & $35 \%$ \\
\hline Time of admission & & \\
\hline AM & 20 & $36 \%$ \\
\hline PM & 32 & $58 \%$ \\
\hline NA & 3 & $5 \%$ \\
\hline Length of Stay & & \\
\hline $1-15$ days & 14 & $25 \%$ \\
\hline $16-30$ days & 22 & $40 \%$ \\
\hline $31-55$ days & 16 & $29 \%$ \\
\hline NA & 3 & $5 \%$ \\
\hline Primary Ref & & $26 \%$ \\
\hline Direct & 26 & $47 \%$ \\
\hline Referred & 17 & $31 \%$ \\
\hline NA & 12 & $22 \%$ \\
\hline Self Ref & 24 & \\
\hline NO & & $34 \%$ \\
\hline YES & & \\
\hline NA & & \\
\hline EMD & & \\
\hline NO & & \\
\hline YES & & \\
\hline NA & & \\
\hline
\end{tabular}

Most of the cases (47\%) are referred directly to the tertiary care hospital while $31 \%$ are referred from the Primary Health Care centers and $22 \%$ referral is not available. While $44 \%$ are self-referred to the neurosurgery dept. $62 \%$ are referred from Emergency to neurosurgery dept. Mostly (58\%) cases are admitted after 12PM, while $36 \%$ are admitted before $12 \mathrm{PM}$ and time of admission for $6 \%$ cases is not available. Most of the patients (40\%) stayed 16-30 days in the hospital (LOS) while 25\% stayed for 1-15 days, $29 \%$ stayed $31-55$ days and LOS for $6 \%$ of cases is not available. [Table 1] Of these 55 referrals, $67 \%$ undergone CT.27\% underwent MRI, $\quad 47 \%$ cases underwent X-ray investigations. It is observed that $2 \%$ undergone all the three i.e., CT, MRI and Xray radio diagnosis while $15 \%$ dint undergo CT, MRI and X-rays. Most of the cases 76\% are brain injuries, $15 \%$ are spinal cord injuries, $5 \%$ are both brain and spinal cord injuries while data of $4 \%$ cases is not available. $69 \%$ are managed surgically in the neurosurgical dept. while $20 \%$ are managed medically and management of the $11 \%$ cases is not available. [Table 2]

Table-2 - Clinical details of the Neurosurgery referral cases

\begin{tabular}{|l|l|l|}
\hline CT & Frequency & Percent \\
\hline YES & 37 & $67 \%$ \\
\hline NO & 18 & $33 \%$ \\
\hline MRI & & \\
\hline YES & 15 & $27 \%$ \\
\hline NO & 40 & $73 \%$ \\
\hline X RAYS & & \\
\hline YES & 4 & $7 \%$ \\
\hline NO & 51 & $93 \%$ \\
\hline Case & & \\
\hline
\end{tabular}




\begin{tabular}{|l|l|l|}
\hline Brain & 42 & $76 \%$ \\
\hline Spinal cord & 8 & $15 \%$ \\
\hline Both & 3 & $5 \%$ \\
\hline NA & 2 & $4 \%$ \\
\hline Investigations & & \\
\hline CT & 37 & $67 \%$ \\
\hline MRI & 15 & $27 \%$ \\
\hline X Rays & 4 & $7 \%$ \\
\hline All & 1 & $2 \%$ \\
\hline None & 8 & $15 \%$ \\
\hline Management & & \\
\hline Medical & 11 & $20 \%$ \\
\hline Surgical & 38 & $69 \%$ \\
\hline NA & 6 & $11 \%$ \\
\hline
\end{tabular}

\section{Discussion}

The data of our study strongly support an urgent need for awareness in the public sector for direct referrals to the neurosurgery dept. within the golden hour and platinum minutes and educating the public with development of a dedicated network of Telemedicine using all sorts of gadgets including android phones for an effective surgical management of the trauma cases from pre-hospital care to reaching early to the tertiary care teaching hospital or well-equipped center of excellence in neurosurgery and neurosciences.

Road trauma is an emergent global issue. There is huge disparity between the population affected by road trauma and the resource allocation. If the current trend continues, a predicted extra five million lives will be lost in this decade and in the younger age groups. India is yet to establish an effective system of delivering trauma care and emergency services to a majority of its citizens despite its economic success. [6]

We have to prepare ourselves urgently as 90 percent of global trauma mortality occurs in low-and-middle-income countries (LMICs). [7] In high income countries (HICs), trauma mortality has steadily declined, but a similar trend is not seen in LMICs. [8] In India, the trend of in-hospital trauma mortality has remained unchanged in the past decade, despite advances in imaging and medical equipment. [9] In addition, India's 30-day trauma mortality rate is twice that of comparable patients admitted to trauma centers in HIC settings. The reasons for the high rates and unchanging trends remain unknown and unexplored. [10] High clinical load [11], low-resources, and high out-of-pocket expenditures [12] are commonly named as barriers to improving trauma care in India. [13] However, several studies have demonstrated that low-cost interventions can improve trauma care outcomes. [7,14] Report on Road Accidents in India 2016, published by Transport Research wing under Ministry of Road Transport \& Highways, Government of India, has revealed that RTAs record at least 413 people died every day in 1,317 road accidents which means that at least 17 deaths occurred in road accidents in 55 accidents every hour in the given time period15 i.e., there is one death on the Indian road every six-eight minutes and this is expected to escalate to one death every three minutes by 2020 . [15]

Though the Neurosurgeons are available in the tertiary care trauma centers, many head injury patients are initially evaluated and 
treated in the primary care centers or nontrauma care centers. It is beyond any discussion that all patients who clearly need a neurosurgical intervention should be transferred as early as possible. However, the inter-hospital referral of trauma case is another complex concern which is to be solved. The decision to transfer a patient to another hospital might definitely influence the decisions in treatment priorities and even the mode of treatment.

The patient demographic profile reflects that most of the trauma victims $47 \%$ are middle aged group 36-55 years and are males $73 \%$ and shows the high prevalence of injuries while attending their duties. Educating and creating awareness regarding the hospital referrals and the importance of timely transfer to the specialized tertiary care trauma centers like $\mathrm{NMCH}$ can result in better evaluation, earlier treatment and best outcomes. There is need to educate health care workers about the quality of care for head and spinal cord injury victims and to ensure early and ongoing neurosurgical care provided in $\mathrm{NMCH}$ and other health care centers. Efforts need to be made at the prehospital level to get victims to the traumatic care centers with in golden hour and provide initial care for which introduction and educating the public with Telemedicine appears to be boon with the team of doctors, nurses, technicians, supporting staff and equipment to be provided to execute the concept of telemedicine. To reduce the burdens of the traumatic injuries, the injury prevention strategies regarding road traffic safety, work place safety, improvement in referral patterns, and implementation of protocol for traumatic registry, quality improvement in pre-hospital, primary health care centers, emergency, neurosurgery, and rehabilitation care are essential.

\section{Strengths and Limitations}

We had done health service research on referral of TBI cases that need holistic approach in this part of country where impediment delay TBI cases to reach devoted neurosurgical intensive care to optimize reduction of case fatality, morbidity and disability. We had several limitations. We had to study referral pattern with a small sample in a single center in the infrastructure poor set up in south-India.

\section{Conclusions}

The study addresses that majority of TBI patients were referred from the site of injury and emergency department depending on time of accident that determined the subsequent investigations and interventions. The analysis strongly suggests that improvement of care for patients with severe head injury represents the best strategy for reduction of case fatality in those hospitalized after blunt trauma and that neurological intensive care intervention is central to such a strategy. The results support an urgent need for awareness in the public sector for direct referrals to the neurosurgery dept. within the golden hour and platinum minutes and educating the public with Telemedicine for an effective surgical management of the trauma cases in the tertiary care teaching hospital which is a center of excellence in neurosurgery and neurosciences. 


\section{Correspondence}

Dr. Amit Agrawal

Professor of Neurosurgery

Department of Neurosurgery

Narayana Medical College Hospital

Chinthareddypalem

Nellore-524003

Andhra Pradesh (India)

Email-dramitagrawal@gmail.com

Mobile- +91-8096410032

\section{References}

1. Peden M, Hyder A. Road traffic injuries are a global public health problem. Bmj 2002;324:1153-1153.

2. Bhalla K, Shotten M, Cohen A, et al. Transport for health: the global burden of disease from motorized road transport2014.

3. Nantulya VM, Reich MR. The neglected epidemic: road traffic injuries in developing countries. BMJ: British Medical Journal 2002;324:1139.

4. Organization WH. Global status report on road safety: time for action: World Health Organization, 2009.

5. WHO. Road traffic injuries [online]. Available at: http://www.who.int/mediacentre/factsheets/fs358/en/.

Accessed 2017-12-13 04:30:54

6. McIntosh B, Sheppy B, Rane S. An Indian tragedy, an Indian solution: perspective of managing service quality in emergency medical services in India. The Journal of Global Health Care Systems 2012;2.

7. Chandran A, Hyder AA, Peek-Asa C. The global burden of unintentional injuries and an agenda for progress. Epidemiologic reviews 2010;32:110-120.
8. Mock C, Joshipura M, Arreola-Risa C, Quansah R. An estimate of the number of lives that could be saved through improvements in trauma care globally. World journal of surgery 2012;36:959-963.

9. Gerdin M, Roy N, Dharap S, et al. Early hospital mortality among adult trauma patients significantly declined between 1998-2011: three single-centre cohorts from Mumbai, India. PloS one 2014;9:e90064.

10. Roy N, Gerdin M, Ghosh S, et al. 30-Day In-hospital trauma mortality in four urban university hospitals using an Indian Trauma Registry. World journal of surgery 2016;40:1299-1307.

11. Stelfox HT, Joshipura M, Chadbunchachai W, et al. Trauma quality improvement in low and middle income countries of the Asia-Pacific region: a mixed methods study. World journal of surgery 2012;36:1978-1992.

12. Kumar GA, Dilip TR, Dandona L, Dandona R. Burden of out-of-pocket expenditure for road traffic injuries in urban India. BMC health services research 2012;12:285.

13. Stewart BT, Quansah R, Gyedu A, Ankomah J, Donkor P, Mock C. Strategic assessment of trauma care capacity in Ghana. World journal of surgery 2015;39:2428-2440.

14. Hashmi ZG, Haider AH, Zafar SN, et al. Hospitalbased trauma quality improvement initiatives: first step toward improving trauma outcomes in the developing world. Journal of Trauma and Acute Care Surgery 2013;75:60-68.

15. Desk EW. Road accidents in India, 2016: 17 deaths on roads every hour, Chennai and Delhi most dangerous. 2017. 